\title{
The homogeneous transfinite diameter of a compact subset of $\mathbb{C}^{N}$
}

\author{
by MieczysŁaW JjDRZEJOWski (Kraków)
}

Abstract. Let $K$ be a compact subset of $\mathbb{C}^{N}$. A sequence of nonnegative numbers defined by means of extremal points of $K$ with respect to homogeneous polynomials is proved to be convergent. Its limit is called the homogeneous transfinite diameter of $K$. A few properties of this diameter are given and its value for some compact subsets of $\mathbb{C}^{N}$ is computed.

1. Introduction. Let $K$ be a compact subset of $\mathbb{C}^{N}$. For a nonnegative integer $s$ let

$$
h_{s}:=\left(\begin{array}{c}
s+N-1 \\
N-1
\end{array}\right) .
$$

Let $e_{s, 1}(z), \ldots, e_{s, h_{s}}(z)$ be all monomials $z^{\alpha}:=z_{1}^{\alpha_{1}} \ldots z_{N}^{\alpha_{N}}$ of degree $s$ ordered lexicographically.

For an integer $k\left(1 \leq k \leq h_{s}\right)$ let $x^{(k)}=\left\{x_{1}, \ldots, x_{k}\right\}$ be a system of $k$ points in $\mathbb{C}^{N}$. Define the "homogeneous Vandermondian" $W_{s}\left(x^{(k)}\right)$ of the system $x^{(k)}$ by

$$
W_{s}\left(x^{(k)}\right):=\operatorname{det}\left[e_{s, i}\left(x_{j}\right)\right]_{i, j=1, \ldots, k} .
$$

Then $W_{s}\left(x^{(k)}\right)$ is a polynomial in $x_{1}, \ldots, x_{k}$ of degree $s k$. Let

$$
W_{s, k}:=\sup \left\{\left|W_{s}\left(x^{(k)}\right)\right|: x^{(k)} \subset K\right\} .
$$

A system $x^{(k)}$ of $k$ points in $K$ is called a system of extremal points of $K$ with respect to homogeneous polynomials if

$$
\left|W_{s}\left(x^{(k)}\right)\right|=W_{s, k} .
$$

In this paper we prove that for every compact subset $K$ of $\mathbb{C}^{N}$ the limit

$$
D(K):=\lim _{s \rightarrow \infty}\left(W_{s, h_{s}}\right)^{1 /\left(s h_{s}\right)}
$$

exists. We call it the homogeneous transfinite diameter of $K$. 
This result gives a positive answer to a question put in [11] (see also [12], p. 93). It is obvious that the limit exists for $N=1$. For $N=2$ the convergence was proved by Leja [4] (see also [5], p. 261). The limit is then equal to $\sqrt{2 \Delta(K)}$, where $\Delta(K)$ is the triangular ecart of $K$.

We also prove a few properties of $D(K)$ (e.g. comparison of $D(K)$ with some other constants connected with $K)$. Using a characterization of $D(K)$ in terms of directional Chebyshev constants, we compute $D(K)$ for

$$
K:=\left\{\left(z_{1}, \ldots, z_{N}\right) \in \mathbb{C}^{N}:\left|z_{1}\right|^{p_{1}}+\ldots+\left|z_{N}\right|^{p_{N}} \leq M\right\},
$$

where $M, p_{1}, \ldots, p_{N}$ are real positive constants.

We also indicate another method for computing $D(K)$ without calculating $W_{s, h_{s}}$.

2. Preliminaries. Let $K$ be a compact subset of $\mathbb{C}^{N}$. Let $\|f\|_{K}$ denote the supremum norm of a function $f: K \rightarrow \mathbb{C}$.

Definition 2.1. $K$ is called unisolvent with respect to homogeneous polynomials if no nonzero homogeneous polynomial vanishes identically on $K$.

Definition 2.2. $K$ is called circled if

$$
\left\{\left(e^{i \theta} z_{1}, \ldots, e^{i \theta} z_{N}\right):\left(z_{1}, \ldots, z_{N}\right) \in K, \quad \theta \in \mathbb{R}\right\} \subset K .
$$

Definition 2.3. $K$ is called $N$-circular if

$$
\left\{\left(e^{i \theta_{1}} z_{1}, \ldots, e^{i \theta_{N}} z_{N}\right):\left(z_{1}, \ldots, z_{N}\right) \in K, \theta_{1}, \ldots, \theta_{N} \in \mathbb{R}\right\} \subset K .
$$

Definition 2.4. Let $\mu$ be a nonnegative Borel measure with supp $\mu \subset$ $K$. The pair $(K, \mu)$ is said to satisfy the Bernstein-Markov property if for every $\lambda>1$ there exists an $M>0$ such that for all polynomials $p$

$$
\|p\|_{K} \leq M \lambda^{\operatorname{deg} p}\|p\|_{2}, \quad \text { where }\|p\|_{2}:=\left(\int_{K}|p|^{2} d \mu\right)^{1 / 2} \text {. }
$$

Remark. A few examples of pairs satisfying the Bernstein-Markov property can be found e.g. in [2], [7], [9], [13].

Let $\delta$ denote the Lebesgue surface area measure on the unit sphere

$$
S:=\left\{z \in \mathbb{C}^{N}:\left|z_{1}\right|^{2}+\ldots+\left|z_{N}\right|^{2}=1\right\},
$$

normalized so that $\int_{S} d \delta=1$.

Definition 2.5 (see [1]). The Alexander constant $\gamma(K)$ is

$$
\gamma(K):=\inf _{s \in \mathbb{N}}\left(\gamma_{s}(K)\right)^{1 / s}=\lim _{s \rightarrow \infty}\left(\gamma_{s}(K)\right)^{1 / s},
$$


where $\gamma_{s}(K):=\inf \left\{\|Q\|_{K}\right\}$, the infimum being taken over all homogeneous polynomials $Q$ of $N$ complex variables of degree $s$, normalized so that

$$
\int_{S} \log |Q|^{1 / s} d \delta=\kappa_{N}:=\int_{S} \log \left|z_{N}\right| d \delta .
$$

It is known that

$$
\kappa_{N}=-\frac{1}{2}\left(1+\frac{1}{2}+\ldots+\frac{1}{N-1}\right) .
$$

Definition 2.6 (see [12]). The Chebyshev constant $\varrho(K)$ is

$$
\varrho(K):=\inf _{s \in \mathbb{N}}\left(\varrho_{s}(K)\right)^{1 / s}=\lim _{s \rightarrow \infty}\left(\varrho_{s}(K)\right)^{1 / s},
$$

where $\varrho_{s}(K):=\inf \left\{\|Q\|_{K}\right\}$, the infimum being taken over all homogeneous polynomials $Q$ of $N$ complex variables of degree $s$, normalized so that $\|Q\|_{S}=1$.

3. The transfinite diameter of a compact subset $K$ of $\mathbb{C}^{N}$. For a nonnegative integer $s$ put

$$
m_{s}:=\left(\begin{array}{c}
s+N \\
N
\end{array}\right) .
$$

Let $e_{1}(z), e_{2}(z), \ldots$ be all monomials $z^{\alpha}:=z_{1}^{\alpha_{1}} \ldots z_{N}^{\alpha_{N}}$ ordered so that the degrees of the $e_{j}(z)$ are nondecreasing and the monomials of a fixed degree are ordered lexicographically. It is easy to check that $e_{s+1, k}=e_{m_{s}+k}$.

For an integer $k$ let $x^{(k)}=\left\{x_{1}, \ldots, x_{k}\right\}$ be a system of $k$ points in $\mathbb{C}^{N}$. Define the "Vandermondian" $V\left(x^{(k)}\right)$ of the system $x^{(k)}$ by

$$
V\left(x^{(k)}\right):=\operatorname{det}\left[e_{i}\left(x_{j}\right)\right]_{i, j=1, \ldots, k} .
$$

Then $V\left(x^{\left(m_{s}\right)}\right)$ is a polynomial in $x_{1}, \ldots, x_{m_{s}}$ of degree

$$
l_{s}:=\sum_{j=1}^{m_{s}} \operatorname{deg} e_{j}=\sum_{k=0}^{s} k h_{k}
$$

It is easy to prove that $l_{s}=N\left(\begin{array}{l}s+N \\ N+1\end{array}\right)$. Put

$$
V_{k}:=\sup \left\{\left|V\left(x^{(k)}\right)\right|: x^{(k)} \subset K\right\} .
$$

Zakharyuta proved in [14] that for every compact subset $K$ of $\mathbb{C}^{N}$ the limit

$$
d(K):=\lim _{s \rightarrow \infty}\left(V_{m_{s}}\right)^{1 / l_{s}}
$$

exists; it is called the transfinite diameter of $K$. This result gave a positive answer to a question put in [6]. For $N=1$ the convergence was proved by Fekete [3] (see also [5]). 
Zakharyuta also computed $d(K)$ in terms of the directional Chebyshev constants. Put

$$
\begin{aligned}
& \Sigma=\Sigma^{N-1}:=\left\{\theta=\left(\theta_{1}, \ldots, \theta_{N}\right) \in \mathbb{R}^{N}: \sum_{j=1}^{N} \theta_{j}=1, \theta_{j} \geq 0\right\}, \\
& \Sigma_{0}=\Sigma_{0}^{N-1}:=\left\{\theta \in \Sigma^{N-1}: \theta_{j}>0 \text { for } j=1, \ldots, N\right\} .
\end{aligned}
$$
Let

For an integer $j \geq 1$ let $\alpha(j):=\left(\alpha_{1}, \ldots, \alpha_{N}\right)$, where $z_{1}^{\alpha_{1}} \ldots z_{N}^{\alpha_{N}}=e_{j}(z)$.

$$
M_{j}:=\inf \left\{\left\|e_{j}(z)+\sum_{i<j} c_{i} e_{i}(z)\right\|_{K}: c_{i} \in \mathbb{C}\right\}
$$

denote the Chebyshev constant of $K$ associated to the monomial $e_{j}(z)$ and the given ordering. It is known that the infimum is attained for at least one polynomial $t_{j}(z)=e_{j}(z)+\sum_{i<j} c_{i} e_{i}(z)$. It is called the Chebyshev polynomial of $K$. Put

$$
\tau_{j}:=M_{j}^{1 /|\alpha(j)|},
$$

where, as usual, $|\alpha(j)|=\alpha_{1}+\ldots+\alpha_{N}$ is the length of the multiindex $\alpha(j)$.

For $\theta \in \Sigma$ let $\tau(K, \theta)$ and $\tau_{-}(K, \theta)$ denote the "Chebyshev constants in the direction $\theta$ ", i.e.

$$
\begin{aligned}
\tau(K, \theta) & :=\lim \sup \left\{\tau_{j}: j \rightarrow \infty, \quad \alpha(j) /|\alpha(j)| \rightarrow \theta\right\}, \\
\tau_{-}(K, \theta) & :=\liminf \left\{\tau_{j}: j \rightarrow \infty, \quad \alpha(j) /|\alpha(j)| \rightarrow \theta\right\} .
\end{aligned}
$$

Zakharyuta proved that $\tau(K, \theta)=\tau_{-}(K, \theta)$ for each $\theta \in \Sigma_{0}$ and that $\log \tau(K, \theta)$ is a convex function on $\Sigma_{0}$. Let

$$
\tau(K):=\exp \left\{\frac{1}{\operatorname{mes} \Sigma} \int_{\Sigma} \log \tau(K, \theta) d \omega(\theta)\right\},
$$

where mes $\Sigma:=\int_{\Sigma} d \omega(\theta)$ and $\omega$ denotes the Lebesgue surface area measure on the hyperplane $\left\{\theta_{1}+\ldots+\theta_{N}=1\right\}$ in $\mathbb{R}^{N}$. Zakharyuta proved that $d(K)=\tau(K)$.

4. The homogeneous transfinite diameter. For two integers $s, k$ $\left(s \geq 0,1 \leq k \leq h_{s}\right)$ put

$$
M_{s, k}:=\inf \left\{\left\|e_{s, k}(z)+\sum_{i<k} c_{i} e_{s, i}(z)\right\|_{K}: c_{i} \in \mathbb{C}\right\} .
$$

It is easy to check that there exists at least one homogeneous polynomial $t_{s, k}(z)=e_{s, k}(z)+\sum_{i<k} c_{i} e_{s, i}(z)$ attaining the infimum. It is called the Chebyshev polynomial of $K$.

Let $\beta(s, k):=\alpha\left(m_{s-1}+k\right)$, where $m_{-1}:=0$. Hence $\beta(s, k)=$ $\left(\beta_{1}, \ldots, \beta_{N}\right)$, where $z_{1}^{\beta_{1}} \ldots z_{N}^{\beta_{N}}=e_{s, k}(z)$. It is obvious that $|\beta(s, k)|=s$. 
Put

For $\theta \in \Sigma$ let

$$
\tau_{s, k}:=M_{s, k}^{1 / s}
$$

$$
\begin{aligned}
\widetilde{\tau}(K, \theta) & :=\lim \sup \left\{\tau_{s, k}: s \rightarrow \infty, \beta(s, k) / s \rightarrow \theta\right\}, \\
\widetilde{\tau}_{-}(K, \theta) & :=\liminf \left\{\tau_{s, k}: s \rightarrow \infty, \beta(s, k) / s \rightarrow \theta\right\} .
\end{aligned}
$$

It is clear that $\widetilde{\tau}(K, \theta) \leq C$ if

$$
K \subset\left\{z \in \mathbb{C}^{N}:\left|z_{1}\right| \leq C, \ldots,\left|z_{N}\right| \leq C\right\} .
$$

The following lemmas can be proved in the same manner as the similar results in [14] (it suffices to replace the polynomials $e_{j}(z)+\sum_{i<j} c_{i} e_{i}(z)$ by $e_{s, k}(z)+\sum_{i<k} b_{i} e_{s, i}(z)$, where $\left.e_{s, k}=e_{j}\right)$ :

LEMMA 4.1. For each $\theta \in \Sigma_{0}, \widetilde{\tau}(K, \theta)=\widetilde{\tau}_{-}(K, \theta)$.

LEMMA 4.2. The function $\log \widetilde{\tau}(K, \theta)$ is convex in $\Sigma_{0}$.

Corollary 4.3. If $\widetilde{\tau}\left(K, \theta^{\prime}\right)=0$ for some $\theta^{\prime} \in \Sigma_{0}$, then $\widetilde{\tau}(K, \theta) \equiv 0$ in $\Sigma_{0}$.

Corollary 4.4. The function $\log \widetilde{\tau}(K, \theta)$ is continuous in $\Sigma_{0}$.

LEMMA 4.5. If $\theta \in \Sigma \backslash \Sigma_{0}$, then

$$
\widetilde{\tau}_{-}(K, \theta)=\liminf \left\{\widetilde{\tau}\left(K, \theta^{\prime}\right): \theta^{\prime} \rightarrow \theta, \theta^{\prime} \in \Sigma_{0}\right\} .
$$

\section{Corollary 4.6.}

$$
\begin{aligned}
\limsup _{s \rightarrow \infty} \tau_{s, k} & =\sup \{\widetilde{\tau}(K, \theta): \theta \in \Sigma\}, \\
\liminf _{s \rightarrow \infty} \tau_{s, k} & =\inf \{\widetilde{\tau}(K, \theta): \theta \in \Sigma\} \\
& =\inf \left\{\widetilde{\tau}(K, \theta): \theta \in \Sigma_{0}\right\}=\inf \left\{\widetilde{\tau}_{-}(K, \theta): \theta \in \Sigma\right\} .
\end{aligned}
$$

Corollary 4.7. If $\widetilde{\tau}(K, \theta) \not \equiv 0$ in $\Sigma_{0}$, then $\inf \{\widetilde{\tau}(K, \theta): \theta \in \Sigma\}>0$.

DeFinition 4.8. The Chebyshev constant $\widetilde{\tau}(K)$ is

$$
\widetilde{\tau}(K):=\exp \left\{\frac{1}{\operatorname{mes} \Sigma} \int_{\Sigma} \log \widetilde{\tau}(K, \theta) d \omega(\theta)\right\} .
$$

If $\widetilde{\tau}(K, \theta) \equiv 0$ in $\Sigma_{0}$, then $\widetilde{\tau}(K)=0$. Assume that $\widetilde{\tau}(K, \theta) \not \equiv 0$ in $\Sigma_{0}$. Then $\log \widetilde{\tau}(K, \theta)$ is continuous in $\Sigma_{0}$ and bounded on $\Sigma$ (see Corollaries 4.4 and 4.7). Therefore the integral above exists and is finite. Hence $0<$ $\widetilde{\tau}(K)<\infty$ in this case.

LEMMA 4.9. $\lim _{s \rightarrow \infty} \widetilde{\tau}_{s}^{0}(K)=\widetilde{\tau}(K)$, where

$$
\widetilde{\tau}_{s}^{0}(K):=\left(\prod_{k=1}^{h_{s}} \tau_{s, k}\right)^{1 / h_{s}} .
$$


LEMMA 4.10. Let $s, k$ be nonnegative integers such that $1 \leq k \leq h_{s}$. Then

$$
\tau_{s, k}^{s} W_{s, k-1} \leq W_{s, k} \leq k \tau_{s, k}^{s} W_{s, k-1}
$$

where $W_{s, 0}:=1$.

Corollary 4.11. If $W_{s, k}>0$ for each $k \in\left\{1, \ldots, h_{s}\right\}$, then

$$
\left(\widetilde{\tau}_{s}^{0}(K)\right)^{s h_{s}} \leq W_{s, h_{s}} \leq h_{s} !\left(\widetilde{\tau}_{s}^{0}(K)\right)^{s h_{s}} .
$$

THEOREM 4.12. For every compact subset $K$ of $\mathbb{C}^{N}$ the limit

$$
D(K):=\lim _{s \rightarrow \infty}\left(W_{s, h_{s}}\right)^{1 /\left(s h_{s}\right)}
$$

exists and is equal to $\widetilde{\tau}(K)$.

We call this limit the homogeneous transfinite diameter of $K$.

Proof. If $K$ is not unisolvent with respect to homogeneous polynomials then $Q \equiv 0$ on $K$, where $Q=e_{s, k}+\sum_{i<k} c_{i} e_{s, i}$. Hence for each positive integer $j$

$$
z_{1}^{j} \ldots z_{N}^{j} Q\left(z_{1}, \ldots, z_{N}\right) \equiv 0 \quad \text { on } K .
$$

Letting $j \rightarrow \infty$ we obtain $\widetilde{\tau}\left(K, \theta^{\prime}\right)=0$, where $\theta^{\prime}=(1 / N, \ldots, 1 / N)$. By Corollary $4.3, \widetilde{\tau}(K, \theta) \equiv 0$ on $\Sigma_{0}$. On the other hand, one sees immediately that $W_{r, h_{r}}=0$ for $r \geq s$, which completes the proof in this case.

Assume now that $K$ is unisolvent with respect to homogeneous polynomials. Then $\tau_{s, k}>0$ for $s \geq 0$ and $1 \leq k \leq h_{s}$. So $W_{s, k}>0$ by Lemma 4.10. Applying Lemma 4.9 and Corollary 4.11 we get the desired conclusion.

Corollary 4.13. If $K$ is not unisolvent with respect to homogeneous polynomials, then $D(K)=0$.

\section{Properties of the constant $D(K)$}

Lemma 5.1. For every compact subset $K$ of $\mathbb{C}^{N}, d(K) \leq D(K)$. If $K$ is circled, then $d(K)=D(K)$.

Proof. It is obvious that $\left\|t_{j}\right\|_{K} \leq\left\|t_{s, k}\right\|_{K}$ if $\beta(s, k)=\alpha(j)$, i.e. $e_{s, k}=$ $e_{j}$. By Theorem 4.12 and the equality $d(K)=\tau(K)$, it suffices to show that $\left\|t_{s, k}\right\|_{K} \leq\left\|t_{j}\right\|_{K}$ if $K$ is circled. By the Cauchy inequalities $\left\|t_{j}\right\|_{K} \geq\left\|q_{j}\right\|_{K}$, where $t_{j}=q_{j}+p_{j}, q_{j}$ is homogeneous and $\operatorname{deg} p_{j}<\operatorname{deg} t_{j}\left(\right.$ or $\left.p_{j} \equiv 0\right)$. Obviously, $\left\|q_{j}\right\|_{K} \geq\left\|t_{s, k}\right\|_{K}$, which proves the lemma.

LEMMA 5.2. If $K$ is $N$-circular and $\theta \in \Sigma_{0}$, then

$$
\tau(K, \theta)=\widetilde{\tau}(K, \theta)=\sup \left\{\left|z_{1}\right|^{\theta_{1}} \ldots\left|z_{N}\right|^{\theta_{N}}:\left(z_{1}, \ldots, z_{N}\right) \in K\right\} .
$$


Proof. Clearly, $\left\|t_{j}\right\|_{K} \leq\left\|t_{s, k}\right\|_{K} \leq\left\|e_{j}\right\|_{K}$, where $e_{s, k}=e_{j}$. Since $K$ is $N$-circular, by the Cauchy inequalities $\left\|e_{j}\right\|_{K} \leq\left\|t_{j}\right\|_{K}$. Hence for $\theta \in \Sigma_{0}$

$$
\begin{aligned}
\tau(K, \theta) & =\widetilde{\tau}(K, \theta)=\lim \left\{\left\|e_{j}\right\|_{K}^{1 /|\alpha(j)|}: j \rightarrow \infty, \alpha(j) /|\alpha(j)| \rightarrow \theta\right\} \\
& =\sup \left\{\left|z_{1}\right|^{\theta_{1}} \ldots\left|z_{N}\right|^{\theta_{N}}:\left(z_{1}, \ldots, z_{N}\right) \in K\right\} .
\end{aligned}
$$

which is the desired conclusion.

Lemma 5.3. $D(K)=D(\widehat{K})$, where $\widehat{K}$ is the convex hull of $K$ with respect to homogeneous polynomials, i.e.

$\widehat{K}:=\left\{z \in \mathbb{C}^{N}:|Q(z)| \leq\|Q\|_{K}\right.$ for all homogeneous polynomials $\left.Q\right\}$.

Proof. It suffices to use Theorem 4.12 together with the obvious equality $\widetilde{\tau}(K, \theta)=\widetilde{\tau}(\widehat{K}, \theta)$.

Lemma 5.4. Let $K_{1}=F\left(K_{2}\right)$, where $F\left(z_{1}, \ldots, z_{N}\right):=\left(c_{1} z_{1}, \ldots, c_{N} z_{N}\right)$ for $\left(z_{1}, \ldots, z_{N}\right) \in \mathbb{C}^{N}$ and $c_{1}, \ldots, c_{N} \in \mathbb{C}$. Then

$$
D\left(K_{1}\right)=\left|c_{1} \ldots c_{N}\right|^{1 / N} D\left(K_{2}\right) .
$$

Proof. It is sufficient to compare the constants $W_{s, h_{s}}$ for $K_{1}$ with those for $K_{2}$. The details are left to the reader.

LEMmA 5.5. If $U: \mathbb{C}^{N} \rightarrow \mathbb{C}^{N}$ is a unitary transformation, then $D(U(K))$ $=D(K)$.

Proof. The lemma can be proved in the same way as the similar result $d(U(K))=d(K)($ see $[8])$.

Corollary 5.6. If $A: \mathbb{C}^{N} \rightarrow \mathbb{C}^{N}$ is a linear mapping, then

$$
D(A(K))=|\operatorname{det} A|^{1 / N} D(K) .
$$

Proof. Combine Lemmas 5.4 and 5.5.

TheOREM 5.7. If $K$ is compact and $R$ is a positive constant such that

$$
K \subset B_{R}:=\left\{\left(z_{1}, \ldots, z_{N}\right) \in \mathbb{C}^{N}:\left|z_{1}\right|^{2}+\ldots+\left|z_{N}\right|^{2} \leq R^{2}\right\},
$$

then

$$
\varrho(K) / \sqrt{N} \leq D(K) \leq R^{1-1 / N} \varrho(K)^{1 / N} .
$$

Proof. The theorem can be proved in the same manner as Theorem 3 in [8] (it suffices to replace $e_{j}(z)+\sum_{i<j} c_{i} e_{i}(z)$ by $e_{s, k}(z)+\sum_{i<k} b_{i} e_{s, i}(z)$, where $e_{s, k}=e_{j}$ ).

COROLlaRY 5.8. If $K$ is compact and $R$ is a positive constant such that $K \subset B_{R}$, then

$$
\gamma(K) / \sqrt{N} \leq D(K) \leq R^{1-1 / N} \exp \left(-\kappa_{N} / N\right) \gamma(K)^{1 / N} .
$$

Proof. It is known that $\gamma(K) \leq \varrho(K) \leq \gamma(K) \exp \left(-\kappa_{N}\right)$ (see [12], Proposition 12.1). Now apply Theorem 5.7. 
THEOREM 5.9. Let $K$ be a compact subset of $\mathbb{C}^{N}$. Let $\mu$ be a nonnegative Borel measure with $\operatorname{supp} \mu \subset K$. If the pair $(K, \mu)$ satisfies the BernsteinMarkov property and $\mu(K)<\infty$, then

$$
D(K)=\lim _{s \rightarrow \infty}\left(G_{s, h_{s}}\right)^{1 /\left(2 s h_{s}\right)},
$$

where

$$
G_{s, k}:=\operatorname{det}\left\{\left[\int_{K} e_{s, i}(z) \overline{e_{s, j}(z)} d \mu(z)\right]_{i, j=1, \ldots, k}\right\},
$$

for nonnegative integers $s, k\left(k \in\left\{1, \ldots, h_{s}\right\}\right)$.

$\mathrm{Proof}$. If $K$ is not unisolvent with respect to homogeneous polynomials then $D(K)=0$ (see Corollary 4.13). On the other hand, for all but a finite number of integers $r$ there exists a nonzero homogeneous polynomial $Q_{r}$ of degree $r$ that vanishes identically on $K$, say

$$
Q_{r}=\sum_{j=1}^{h_{r}} d_{j} e_{r, j} \quad\left(d_{j} \in \mathbb{C}\right) .
$$

Obviously, $\left\|Q_{r}\right\|_{K}=0$ implies $\left\|Q_{r}\right\|_{2}=0$. Therefore $G_{r, h_{r}}=0$ for such $r$.

Assume that $K$ is unisolvent with respect to homogeneous polynomials. Then none of the Gram determinants $G_{s, k}$ is zero. Indeed, if $G_{s, k}=0$ for some $s$ and $k$, we should have $\|Q\|_{2}=0$, where $Q=\sum_{j=1}^{k} d_{j} e_{s, j}\left(d_{j} \in\right.$ $\mathbb{C})$ and $Q \not \equiv 0$. By the Bernstein-Markov property, $\|Q\|_{K}=0$, which is impossible.

Analysis similar to that in the proof of Theorem 3.3 in [2] now yields our statement (upon replacing again $e_{j}(z)+\sum_{i<j} c_{i} e_{i}(z)$ by $e_{s, k}(z)+$ $\sum_{i<k} b_{i} e_{s, i}(z)$, where $\left.e_{s, k}=e_{j}\right)$. Lemma 4.9 and Theorem 4.12 are used in the proof.

6. The value of $D(K)$ and $d(K)$ for some compact sets $K$. Consider the following compact $N$-circular set $K=K\left(p_{1}, \ldots, p_{N}, M\right)$ :

$$
K:=\left\{\left(z_{1}, \ldots, z_{N}\right) \in \mathbb{C}^{N}:\left|z_{1}\right|^{p_{1}}+\ldots+\left|z_{N}\right|^{p_{N}} \leq M\right\},
$$

where $M, p_{1}, \ldots, p_{N}$ are real positive constants.

Theorem 6.1. If $K=K\left(p_{1}, \ldots, p_{N}, M\right)$ and $a_{j}=1 / p_{j}$ for $j=1, \ldots, N$, then

$D(K)=d(K)=\exp \left\{\frac{1}{N}\left(\sum_{j=1}^{N} a_{j} \log \left(M a_{j}\right)-\frac{1}{2 \pi i} \int_{C} \frac{z^{N} \log z d z}{\left(z-a_{1}\right) \ldots\left(z-a_{N}\right)}\right)\right\}$,

where $C$ is any contour in the right half-plane $\{z \in \mathbb{C}: \operatorname{Re} z>0\}$ enclosing all the points $a_{1}, \ldots, a_{N}$ and $\log z$ is the principal branch of the logarithm. 
In particular, if $p_{j} \neq p_{k}$ for $j \neq k$, then

$$
D(K)=d(K)=\exp \left\{\frac{1}{N}\left(\sum_{j=1}^{N} a_{j} \log \left(M a_{j}\right)-\sum_{j=1}^{N} \frac{a_{j}^{N} \log a_{j}}{\prod_{\substack{k=1 \\ k \neq j}}^{N}\left(a_{j}-a_{k}\right)}\right)\right\} .
$$

If $p_{1}=\ldots=p_{N}=p$ and $M=R^{p}(R>0)$, then

$$
D(K)=d(K)=R \exp \left(-\frac{1}{p} \sum_{k=2}^{N} \frac{1}{k}\right) .
$$

We first prove two lemmas.

Lemma 6.2. If $f\left(\theta_{1}, \ldots, \theta_{N}\right)$ is a continuous function on $\Sigma^{N-1}$, then

$$
\frac{1}{\operatorname{mes} \Sigma^{N-1}} \int_{\Sigma^{N-1}} f\left(\theta_{1}, \ldots, \theta_{N}\right) d \omega(\theta)=(N-1) \int_{0}^{1} x^{N-2} H(x) d x,
$$

where

$$
H(x):=\frac{1}{\operatorname{mes} \Sigma^{N-2}} \int_{\Sigma^{N-2}} f\left(\xi_{1} x, \xi_{2} x, \ldots, \xi_{N-1} x, 1-x\right) d \omega(\xi) .
$$

Pr o of. Obviously,

$$
\begin{aligned}
\frac{1}{\operatorname{mes} \Sigma^{N-1}} & \int_{\Sigma^{N-1}} f\left(\theta_{1}, \ldots, \theta_{N}\right) d \omega(\theta) \\
& =\frac{1}{\operatorname{mes} \Sigma_{*}^{N-1}} \int_{\Sigma_{*}^{N-1}} f\left(\theta_{1}, \ldots, \theta_{N-1}, 1-\sum_{j=1}^{N-1} \theta_{j}\right) d \theta_{1} \ldots d \theta_{N-1},
\end{aligned}
$$

where $\Sigma_{*}^{N-1}:=\left\{\left(\theta_{1}, \ldots, \theta_{N-1}\right) \in \mathbb{R}^{N-1}: \sum_{j=1}^{N-1} \theta_{j} \leq 1, \theta_{j} \geq 0\right\}$. We change the variables:

$$
\begin{gathered}
\theta_{j}=\xi_{j} x \quad \text { for } j=1, \ldots, N-2, \\
\theta_{N-1}=\left(1-\sum_{j=1}^{N-2} \xi_{j}\right) x
\end{gathered}
$$

where $0 \leq x \leq 1$ and $\left(\xi_{1}, \ldots, \xi_{N-2}\right) \in \Sigma_{*}^{N-2}$. It is obvious that $d \theta_{1} \ldots d \theta_{N-1}=x^{N-2} d x d \xi_{1} \ldots d \xi_{N-2}$ and that

$$
\operatorname{mes} \Sigma_{*}^{N-2} / \operatorname{mes} \Sigma_{*}^{N-1}=N-1 \text {. }
$$

This proves the lemma (the details are left to the reader). 
Lemma 6.3. If $a_{j} \neq a_{k}$ for $j \neq k$, then

$$
\begin{aligned}
& \sum_{j=1}^{N} \frac{1}{\prod_{\substack{k=1 \\
k \neq j}}^{N}\left(a_{j}-a_{k}\right)}=0, \\
& \sum_{j=1}^{N} \frac{a_{j}^{N}}{\prod_{\substack{k=1 \\
k \neq j}}^{N}\left(a_{j}-a_{k}\right)}=\sum_{j=1}^{N} a_{j} .
\end{aligned}
$$

Pro of. Consider the polynomial

$$
P(x)=\sum_{m=0}^{N-1} b_{m} x^{m}:=-1+\sum_{j=1}^{N} P_{j}(x),
$$

where

$$
P_{j}(x):=\prod_{\substack{k=1 \\ k \neq j}}^{N} \frac{x-a_{k}}{a_{j}-a_{k}} .
$$

It is clear that $\operatorname{deg} P \leq N-1$ and $P\left(a_{j}\right)=0$ for $j=1, \ldots, N$, which implies $P \equiv 0$. So $b_{N-1}=0$, and (6.1) follows.

To prove (6.2), let

$$
Q(x)=-x^{N}+\sum_{m=0}^{N-1} c_{m} x^{m}:=-x^{N}+\sum_{j=1}^{N} a_{j}^{N} P_{j}(x) .
$$

Since $\operatorname{deg} Q=N$ and $Q\left(a_{j}\right)=0$ for $j=1, \ldots, N$, we have

$$
Q(x)=-\left(x-a_{1}\right)\left(x-a_{2}\right) \ldots\left(x-a_{N}\right) .
$$

Therefore $c_{N-1}=\sum_{j=1}^{N} a_{j}$, which completes the proof.

Proof of Theorem 6.1. It is easy to check, applying Lemma 5.2, that for $K=K\left(p_{1}, \ldots, p_{N}, M\right)$ and $\theta \in \Sigma_{0}$

$$
\begin{aligned}
\log \tau(K, \theta)= & \log \widetilde{\tau}(K, \theta) \\
= & \sum_{j=1}^{N} a_{j} \theta_{j} \log \left(M a_{j}\right)+\sum_{j=1}^{N} a_{j} \theta_{j} \log \theta_{j} \\
& -\sum_{j=1}^{N} a_{j} \theta_{j} \log \left(a_{1} \theta_{1}+\ldots+a_{N} \theta_{N}\right) .
\end{aligned}
$$

Since $D(K)=d(K)=\widetilde{\tau}(K)$, it is sufficient to prove the following three formulas $(j=1, \ldots, N)$ :

$$
\frac{1}{\operatorname{mes} \Sigma^{N-1}} \int_{\Sigma^{N-1}} \theta_{j} d \omega(\theta)=\frac{1}{N},
$$




$$
\begin{aligned}
& \frac{1}{\operatorname{mes} \Sigma^{N-1}} \int_{\Sigma^{N-1}} \theta_{j} \log \theta_{j} d \omega(\theta)=-\frac{1}{N} \sum_{k=2}^{N} \frac{1}{k}, \\
& \frac{1}{\operatorname{mes} \Sigma^{N-1}} \int_{\Sigma^{N-1}}\left(\sum_{j=1}^{N} a_{j} \theta_{j}\right) \log \left(\sum_{j=1}^{N} a_{j} \theta_{j}\right) d \omega(\theta) \\
& =-\frac{1}{N}\left(\sum_{k=2}^{N} \frac{1}{k}\right)\left(\sum_{j=1}^{N} a_{j}\right)+\frac{1}{N} \cdot \frac{1}{2 \pi i} \int_{C} \frac{z^{N} \log z d z}{\left(z-a_{1}\right) \ldots\left(z-a_{N}\right)} .
\end{aligned}
$$

Observe that the particular cases

$$
p_{j} \neq p_{k} \quad \text { for } j \neq k
$$

and

$$
p_{1}=\ldots=p_{N}=p, \quad M=R^{p}
$$

can be obtained from the main formula (it suffices to apply the Residue Theorem and observe that $f^{(N-1)}(z)=N ! z\left(\log z+\frac{1}{2}+\frac{1}{3}+\ldots+\frac{1}{N}\right)$ if $\left.f(z)=z^{N} \log z\right)$.

It suffices to prove (6.3) and (6.4) for $j=N-1$. Obviously mes $\Sigma_{*}^{N-1}=$ $1 /(N-1)$ ! and

$$
\frac{1}{\operatorname{mes} \Sigma^{N-1}} \int_{\Sigma^{N-1}} \theta_{N-1} d \omega(\theta)=\frac{1}{\operatorname{mes} \Sigma_{*}^{N-1}} \int_{\Sigma_{*}^{N-1}} \theta_{N-1} d \theta_{1} \ldots d \theta_{N-1} .
$$

So (6.3) follows immediately if we change the variables:

$$
\begin{aligned}
\theta_{1} & =\left(1-v_{1}\right) v_{2} \ldots v_{N-1}, \\
\theta_{2} & =\left(1-v_{2}\right) v_{3} \ldots v_{N-1}, \\
\vdots & \\
\theta_{N-2} & =\left(1-v_{N-2}\right) v_{N-1}, \\
\theta_{N-1} & =1-v_{N-1},
\end{aligned}
$$

where $0 \leq v_{j} \leq 1$ for $j=1, \ldots, N-1$.

Apply the same change of variables to compute

$$
\frac{1}{\operatorname{mes} \Sigma_{*}^{N-1}} \int_{\Sigma_{*}^{N-1}} \theta_{N-1} \log \theta_{N-1} d \theta_{1} \ldots d \theta_{N-1} .
$$

Then it is sufficient to check that

$$
\int_{0}^{1} x^{N-2}(1-x) \log (1-x) d x=-\frac{1}{N(N-1)} \sum_{k=2}^{N} \frac{1}{k} .
$$


Let $x=1-e^{-t}$. We obtain

$$
\begin{aligned}
-\int_{0}^{\infty} t e^{-2 t} & \left(1-e^{-t}\right)^{N-2} d t \\
& =-\sum_{j=0}^{N-2}\left(\begin{array}{c}
N-2 \\
j
\end{array}\right)(-1)^{j} \int_{0}^{\infty} t e^{-(j+2) t} d t \\
& =-\sum_{j=0}^{N-2}\left(\begin{array}{c}
N-2 \\
j
\end{array}\right)(-1)^{j} \frac{1}{(j+2)^{2}} \\
& =-\frac{1}{N(N-1)} \sum_{j=0}^{N-2}(-1)^{j}\left(\begin{array}{c}
N \\
j+2
\end{array}\right)\left(1-\frac{1}{j+2}\right) \\
& =-\frac{1}{N(N-1)}\left(\sum_{k=2}^{N}(-1)^{k}\left(\begin{array}{c}
N \\
k
\end{array}\right)+\sum_{k=2}^{N}(-1)^{k+1}\left(\begin{array}{c}
N \\
k
\end{array}\right) \frac{1}{k}\right) \\
& =-\frac{1}{N(N-1)}\left(\frac{1}{2}+\frac{1}{3}+\ldots+\frac{1}{N}\right) .
\end{aligned}
$$

We have applied the well-known formula

$$
\sum_{k=1}^{N}(-1)^{k+1}\left(\begin{array}{l}
N \\
k
\end{array}\right) \frac{1}{k}=\sum_{j=1}^{N} \frac{1}{j}
$$

and the obvious equality

$$
0=(1-1)^{N}=1-N+\sum_{k=2}^{N}(-1)^{k}\left(\begin{array}{l}
N \\
k
\end{array}\right)
$$

Let us prove (6.5). Both its sides are continuous functions of the parameters $a_{j}$. Therefore it suffices to show that the formula is true if $a_{j} \neq a_{k}$ for $j \neq k$. So we have to check that

$$
\begin{aligned}
\frac{1}{\operatorname{mes} \Sigma^{N-1}} \int_{\Sigma^{N-1}}\left(\sum_{j=1}^{N} a_{j} \theta_{j}\right) \log \left(\sum_{j=1}^{N} a_{j} \theta_{j}\right) d \omega(\theta) \\
=-\frac{1}{N}\left(\sum_{k=2}^{N} \frac{1}{k}\right)\left(\sum_{j=1}^{N} a_{j}\right)+\frac{1}{N} \sum_{j=1}^{N} \frac{a_{j}^{N} \log a_{j}}{\prod_{\substack{N=1 \\
k \neq j}}^{N}\left(a_{j}-a_{k}\right)} .
\end{aligned}
$$

The proof is by induction on $N$. It is easy to check the case $N=2$. Assuming (6.6) to hold for $N-1(N \geq 3)$, we will prove it for $N$. We are going to 
apply Lemma 6.2. We first compute

$$
\begin{aligned}
\operatorname{mes} & \Sigma^{N-2} \cdot H(x) \\
= & \int_{\Sigma^{N-2}}\left\{a_{N}(1-x)+\sum_{j=1}^{N-1} a_{j} \xi_{j} x\right\} \log \left\{a_{N}(1-x)+\sum_{j=1}^{N-1} a_{j} \xi_{j} x\right\} d \omega(\xi) .
\end{aligned}
$$

We have $a_{N}(1-x) \equiv a_{N}(1-x) \sum_{j=1}^{N-1} \xi_{j}$ on $\Sigma^{N-2}:=\left\{\sum_{j=1}^{N-1} \xi_{j}=1\right\}$. Therefore

$$
\operatorname{mes} \Sigma^{N-2} \cdot H(x)=\int_{\Sigma^{N-2}}\left(\sum_{j=1}^{N-1} A_{j} \xi_{j}\right) \log \left(\sum_{j=1}^{N-1} A_{j} \xi_{j}\right) d \omega(\xi),
$$

where $A_{j}=A_{j}(x):=a_{N}+\left(a_{j}-a_{N}\right) x$ for $j=1, \ldots, N-1$. By assumption,

$$
\begin{aligned}
H(x)= & -\frac{1}{N-1}\left(\sum_{k=2}^{N-1} \frac{1}{k}\right)\left(\sum_{j=1}^{N-1} A_{j}\right)+\frac{1}{N-1} \sum_{j=1}^{N-1} \frac{A_{j}^{N-1} \log A_{j}}{\prod_{\substack{N=1 \\
k \neq 1 \\
k \neq j}}^{N-1}\left(A_{j}-A_{k}\right)} \\
= & -\frac{1}{N-1}\left(\sum_{k=2}^{N-1} \frac{1}{k}\right)\left\{(N-1) a_{N}+\left(\sum_{j=1}^{N-1} a_{j}-(N-1) a_{N}\right) x\right\} \\
& +\frac{1}{N-1} \sum_{j=1}^{N-1} \frac{\left(a_{N}+\left(a_{j}-a_{N}\right) x\right)^{N-1} \log \left(a_{N}+\left(a_{j}-a_{N}\right) x\right)}{\prod_{\substack{k=1 \\
k \neq j}}^{N-1}\left(a_{j}-a_{k}\right) x} .
\end{aligned}
$$

Applying Lemma 6.2 we obtain

$$
\begin{aligned}
& \frac{1}{\operatorname{mes} \Sigma^{N-1}} \int_{\Sigma^{N-1}}\left(\sum_{j=1}^{N} a_{j} \theta_{j}\right) \log \left(\sum_{j=1}^{N} a_{j} \theta_{j}\right) d \omega(\theta) \\
& =(N-1) \int_{0}^{1} x^{N-2} H(x) d x=B_{1}+B_{2}+B_{3},
\end{aligned}
$$

where

$$
\begin{aligned}
& B_{1}=-(N-1)\left(\sum_{k=2}^{N-1} \frac{1}{k}\right) a_{N} \int_{0}^{1} x^{N-2} d x \\
& B_{2}=-\left(\sum_{k=2}^{N-1} \frac{1}{k}\right)\left(\sum_{j=1}^{N-1} a_{j}-(N-1) a_{N}\right) \int_{0}^{1} x^{N-1} d x, \\
& B_{3}=\sum_{j=1}^{N-1} \frac{\int_{0}^{1}\left(a_{N}+\left(a_{j}-a_{N}\right) x\right)^{N-1} \log \left(a_{N}+\left(a_{j}-a_{N}\right) x\right) d x}{\prod_{\substack{N-1 \\
k \neq j \\
k \neq j}}\left(a_{j}-a_{k}\right)} .
\end{aligned}
$$


It is easy to check that

$$
B_{1}+B_{2}=-\frac{1}{N}\left(\sum_{k=2}^{N-1} \frac{1}{k}\right)\left(\sum_{j=1}^{N} a_{j}\right)
$$

Integrating by parts the integral in $B_{3}$, we obtain $B_{3}=C_{1}+C_{2}$, where

$$
\begin{aligned}
C_{1} & =-\frac{1}{N^{2}} \sum_{j=1}^{N-1} \frac{a_{j}^{N}-a_{N}^{N}}{\prod_{\substack{k=1 \\
k \neq j}}^{N}\left(a_{j}-a_{k}\right)}, \\
C_{2} & =\frac{1}{N} \sum_{j=1}^{N-1} \frac{a_{j}^{N} \log a_{j}-a_{N}^{N} \log a_{N}}{\prod_{\substack{k=1 \\
k \neq j}}^{N}\left(a_{j}-a_{k}\right)} .
\end{aligned}
$$

Applying (6.1) and (6.2) we get

$$
C_{1}=-\frac{1}{N^{2}}\left(\sum_{j=1}^{N-1} \frac{a_{j}^{N}}{\prod_{\substack{k=1 \\ k \neq j}}^{N}\left(a_{j}-a_{k}\right)}+\frac{a_{N}^{N}}{\prod_{k=1}^{N-1}\left(a_{N}-a_{k}\right)}\right)=-\frac{1}{N^{2}} \sum_{j=1}^{N} a_{j} .
$$

Therefore

$$
B_{1}+B_{2}+C_{1}=-\frac{1}{N}\left(\sum_{k=2}^{N} \frac{1}{k}\right)\left(\sum_{j=1}^{N} a_{j}\right)
$$

By (6.1),

$$
\begin{aligned}
C_{2} & =\frac{1}{N}\left(\sum_{j=1}^{N-1} \frac{a_{j}^{N} \log a_{j}}{\prod_{\substack{k=1 \\
k \neq j}}^{N}\left(a_{j}-a_{k}\right)}-a_{N}^{N} \log a_{N} \sum_{j=1}^{N-1} \frac{1}{\prod_{\substack{k=1 \\
k \neq j}}^{N}\left(a_{j}-a_{k}\right)}\right) \\
& =\frac{1}{N} \sum_{j=1}^{N} \frac{a_{j}^{N} \log a_{j}}{\prod_{\substack{k=1 \\
k \neq j}}^{N}\left(a_{j}-a_{k}\right)} .
\end{aligned}
$$

Thus $B_{1}+B_{2}+C_{1}+C_{2}$ is equal to the right-hand side of (6.6), which proves the theorem.

Corollary 6.4 (see [10]). If

$$
K:=\left\{\left(z_{1}, \ldots, z_{N}\right) \in \mathbb{C}^{N}:\left|z_{1}\right| \leq R_{1}, \ldots,\left|z_{N}\right| \leq R_{N}\right\},
$$

where $R_{j}>0$ for $j=1, \ldots, N$, then

$$
D(K)=d(K)=\left(\prod_{j=1}^{N} R_{j}\right)^{1 / N}
$$


Proof. It is easy to check, applying Lemma 5.2, that for $\theta \in \Sigma_{0}$

$$
\log \tau(K, \theta)=\log \widetilde{\tau}(K, \theta)=\sum_{j=1}^{N} \theta_{j} \log R_{j} .
$$

Applying Theorem 4.12 and (6.3) we obtain the desired conclusion.

\section{References}

[1] H. Alexander, Projective capacity, in: Conference on Several Complex Variables, Ann. of Math. Stud. 100, Princeton Univ. Press, 1981, 3-27.

[2] T. Bloom, L. Bos, C. Christensen and N. Leven berg, Polynomial interpolation of holomorphic functions in $\mathbb{C}$ and $\mathbb{C}^{n}$, preprint, 1989.

[3] M. Fekete, Über die Verteilung der Wurzeln bei gewissen algebraischen Gleichungen mit ganzzahligen Koeffizienten, Math. Z. 17 (1923), 228-249.

[4] F. Leja, Sur les séries des polynômes homogènes, Rend. Circ. Mat. Palermo 56 (1932), 419-445.

[5] - , Theory of Analytic Functions, PWN, Warszawa 1957 (in Polish).

[6] _- Problèmes à résoudre posés à la Conférence, Colloq. Math. 7 (1959), 153.

[7] N. Levenberg, Monge-Ampère measures associated to extremal plurisubharmonic functions in $\mathbb{C}^{N}$, Trans. Amer. Math. Soc. 289 (1) (1985), 333-343.

[8] N. Levenberg and B. A. Taylor, Comparison of capacities in $\mathbb{C}^{n}$, in: Proc. Toulouse 1983, Lecture Notes in Math. 1094, Springer, 1984, 162-172.

[9] Nguyen Thanh Van, Familles de polynômes ponctuellement bornées, Ann. Polon. Math. 31 (1975), 83-90.

[10] M. Schiffer and J. Siciak, Transfinite diameter and analytic continuation of functions of two complex variables, Technical Report, Stanford 1961.

[11] J. Siciak, On some extremal functions and their applications in the theory of analytic functions of several complex variables, Trans. Amer. Math. Soc. 105 (2) (1962), $322-357$

[12] - Extremal plurisubharmonic functions and capacities in $\mathbb{C}^{n}$, Sophia Kokyuroku in Math. 14, Sophia University, Tokyo 1982.

[13] —, Families of polynomials and determining measures, Ann. Fac. Sci. Toulouse 9 (2) (1988), 193-211.

[14] V. P. Zakharyuta, Transfinite diameter, Chebyshev constants and a capacity of a compact set in $\mathbb{C}^{n}$, Mat. Sb. 96 (138) (3) (1975), 374-389 (in Russian).

INSTITUTE OF MATHEMATICS

JAGIELLONIAN UNIVERSITY

REYMONTA 4

30-059 KRAKÓW, POLAND 